\title{
Review on magneto-rheological fluid and its application
}

\author{
Xu Biao, Luo Yiping, Ren Hongjuan \\ College of Automobile Engineering, Shanghai University of Engineering Science, Shanghai 201620, China \\ Email address: \\ xubiao0813@163.com (Xu biao), lyp777@sina.com (Luo Yiping), ren-hongjuan@163.com (Ren Hongjuan)
}

To cite this article:

Xu Biao, Luo Yiping, Ren Hongjuan. Review on Magneto-Rheological Fluid and its Application. American Journal of Nanoscience and Nanotechnology. Vol. 2, No. 4, 2014, pp. 70-74. doi: 10.11648/j.nano.20140204.12

\begin{abstract}
Magnetorheological fluids have received widespread attention as smart materials due to their tunable properties. MRF consists of three key components including, soft magnetic particles, carrier liquids, and additives. This review details the characteristics, composition, and rheological principle of MRF, working principle of MRF devices, and their applications in other engineering arenas. The aim is to provide a basis understanding of magneto-rheological components and technology to the readers.
\end{abstract}

Keywords: Magnetorheological Fluid, Application, Working Principle, Components

\section{Introduction}

Magneto-rheological fluid, calls MRF, is a new branch of smart materials[1]. It is a kind of suspension that consists of magnetic particles and carrier fluid and its rheological property can be changed rapidly and reversibly under the action of an external magnetic field, otherwise, it can realize the real-time active control[2]. Magneto-rheological fluid has the extremely widespread application prospect in many fields including aerospace, automotive industry, hydraulic transmission, biotechnology and medical because of its characteristics of continuous, reversible, rapid and easy control[3-6].

\section{Composition and Principle of MRF}

\subsection{Composition of Magneto-Rheological Fluid}

Typical magneto-rheological fluid consists of 3 parts: soft magnetic particles, the carrier fluids and additives[7].

Soft magnetic particles mainly including $\mathrm{Fe}_{3} \mathrm{O}_{4} 、 \mathrm{Fe}_{3}$ 、 Fe、Co、Ni etc.. Among them iron cobalt alloy has the highest magnetic saturation and its magnetic saturation can reach $2.4 \mathrm{~T}$, but it is expensive. In fact, the most widely used materials is pure iron and carbonyl iron powder, their magnetic saturation can reach approximately $2.1 \mathrm{~T}$, other metals, alloys and oxide particles' magnetic saturation is lower than iron. Magnetic particles' diameter is $0.1 \sim 100$ um, the typical value is $3 \sim 5 \mathrm{um}$, only the oxide particles' diameter reach $30 \mathrm{~nm}$, but the magnetic saturation of this oxide particles is relatively low and the maximum stress can reach $5 \mathrm{kPa}$ of corresponding $\mathrm{MRF}[8]$.

The carrier liquid is an important component of magneto-rheological fluid, it is a media of soft magnetic particles suspension. Synthetic oil, Mineral oil and water etc. can be carrier liquid. The carrier liquid of magneto-rheological fluid should have the following characteristics: (1)high boiling point and low freezing point. This ensures that the magneto-rheological fluid has a wide operating range of working temperature; (2) suitable viscosity. Zero magnetic field of MRF should have low viscosity, the lower viscosity, the better characteristics of carrier liquid. But the sedimentation stability becomes worse as the viscosity reducing.

Additives include dispersant and anti-sedimentation agent etc. The main function of additives is to improve the sedimentation stability, dispersion, zero viscosity and shear yield strength of magneto-rheological fluid. Dispersant includes oleic acid and oleic acid salt, naphthenate, sulfonate, phosphate, stearic, glycerol monooleate, alcohols and silica, etc. Anti-sedimentation agent includes high polymer, hydrophilic silicone oligomer, organometallic silicon copolymer, ultrafine amorphous silica, organoclay and oligomer containing hydrogen, etc[9].

\subsection{Principle of Magnetic-Rheological Fluid}

Micro particles are magnetized to produce orderly movement when an external magnetic field is applied, this movement generating at the beginning of micro particles are magnetized and finishing until reaching a relatively stable 
state, forming a fixed structure. These particles are attracted to each other under the force of the magnetic field forming a chain structure, this process is called chain process, as shown in figure 1. [10] These magnetic micro particles are seen as some of rigid sphere.

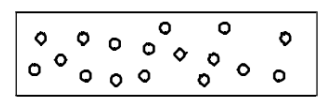

(1)

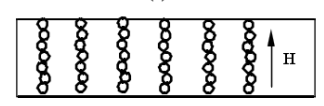

(3)

0000000

0.0000000

Figure 1. Principle of magnetic-rheological fluid

Figure 1 (1) indicates the micro particles disperses irregularly in the carrier liquid when there is no magnetic field, and this time dynamic yield stress is zero. Figure 1(2) indicates that under the magnetic field, micro-particle forms dipole and gets magnetized. Figure 1 (3) indicates the micro particles in MRF connecting into chains under the action of medium magnetic field and then the viscosity of MRF increases. Figure 1 (4) indicates MRF presents obvious magneto-rheological effect: number of chains increases, diameter of chains thicken, dynamic yield stress and apparent viscosity of the magneto-rheological fluid continues to increase. Figure 1(5) indicates that when the external magnetic field is removed, the MRF materials recover quickly and return to the original state of irregular dispersion. This entire chain process takes only a few milliseconds.

\section{Application in Damping}

According to the characteristics of MRF rheological properties in the magnetic field, MRF dampers can adjust itself damping in real time according to external vibration to achieve the purpose of damping. So the MRF damper is an important field of MRF application in engineering.

Its working principle is shown in figure 2.

When MRF damping work properly, the coil produces the magnetic field, magnetic field line acts on magneto-rheological fluid which is in the multiple circular damp channels on the baffle, the piston to do reciprocating linear motion in the cylinder body changing the motion characteristics of MRF in the annular damping channel and changing the pressure difference between the upper and lower chambers, and then achieving the controlling to damping force.

The Maryland University designed a two fold annular gap throttle MRF damper for artillery recoil device, Yang Guangqiang et al [11] tested the damping force performance of $200 \mathrm{kN}$ magneto-rheological damper systematically; Nevada University research team CIML laboratory led by
Professor Gordaninejad developed a double-piston magnetic magneto-rheological fluid damper[12]; Li Zhongxian et al [13] designed and produced double rod shear valve magneto-rheological damper; Wang Hongtao et al [14] designed a disc-sharped slit binoculars magneto-rheological fluid damper based on passive tube shock absorber; Wang Xiuyong et al [15] designed and produced rotary shear MR damper which damping force adjustable range is large and displacement is not restricted.

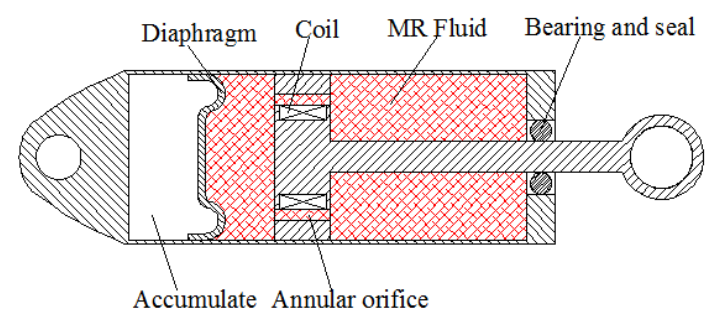

Figure 2. Principle of magnetic-rheological fluid damping

\section{Application in Transmission}

Magneto-rheological transmission was a new transmission technology developing in the 1990s, the transmission theory is based on the rheological effect of MRF. Its working principle is shown in Figure 3, magneto-rheological fluid is the transmission medium, magnetic field generates by the coil acting on the MRF between the turntable and the housing, the input shaft drives the turntable and then rubs the magneto-rheological fluid, by adjusting the magnetic field intensity to change the magneto-rheological fluid shear yield stress, thereby changing the value of the transmission torque and force[16].

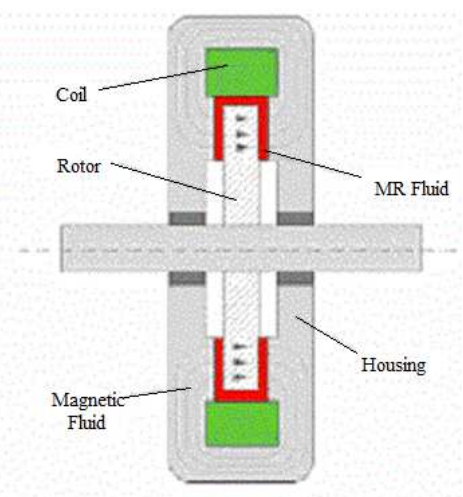

Figure 3. principle of magnetic-rheological fluid transmission

Domestic and foreign scholars do a lot of research on the magneto rheological fluid transmission device. Zheng Jun in Chongqing University[17] developed a cylindrical magneto-rheological fluid actuator, discussed the MRF transmission fluid dynamics, studied the flow field in the steady and transient conditions and explored the response time and influencing factors of MRF transmission device. Hou Youfu etc. in China University of Mining Technology[18] studied the power transmission mechanism 
of MRF between the rotating interface, applied for the related patent about MRF actuator and development small power actuator; Ding Boqun etc. in Northeast Forestry University[19] designed an automobile within wheel impeller MRF brake and deduce its braking torque calculation. This brake braking torque can meet the needs of the general small car.

\section{Application in Hydraulic System}

Magneto-rheological fluid is the working medium of the hydraulic system, under the control of MRF valve it can complete the action of the implementation drive. In this system, the MRF valve is a proportional control valve without moving, shown in Figure 4. Compared with the conventional hydraulic proportional valve, MRF valve has longer life and cheaper price.

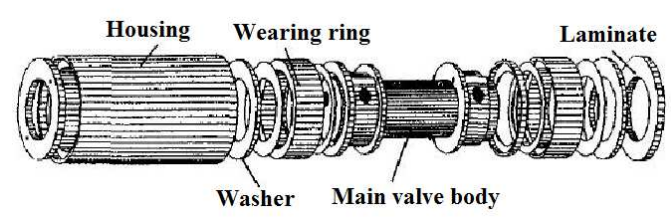

Figure 4. Magnetic-rheological fluid hydraulic valve

Kordonsky [20] proposed a hydraulic cylinder piston motion control scheme and designed variety of structures compact, channel-optimized MRF valves and systems. Magneto-rheological principle of hydraulic system is shown in figure 5, valve resistance changes when the coil of the corner two pairs of hydraulic valves is energized causing the piston-cylinder generates pressure differential, and then the piston moves. This system can locate the actuator.

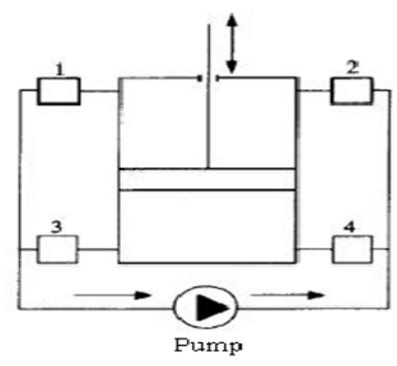

Figure 5. Magnetic-rheological fluid hydraulic system

\section{Application in Polishing Device}

W.I. Kordonski, L.V. Prokhorov etc. [21] combined the theory of electromagnetism and fluid dynamics and invented polishing technology. MRF basic principle is shown in Figure 6, we may even just take the Polishing spherical surface device as an example, puts the MRF on the rotating tray, the workpiece is mounted on a rotating shaft, rotating tray sends the MRF to the workpiece surface, The MRF will stiffen and be dragged into the gap between the workpiece and tray when DC magnetic field is applied in the vicinity. The workpiece is polished because shear in the area becomes high. Workpiece surface sweeps high shear stress area since shaft deflection controlled by the program, therefore, the entire workpiece is polished.

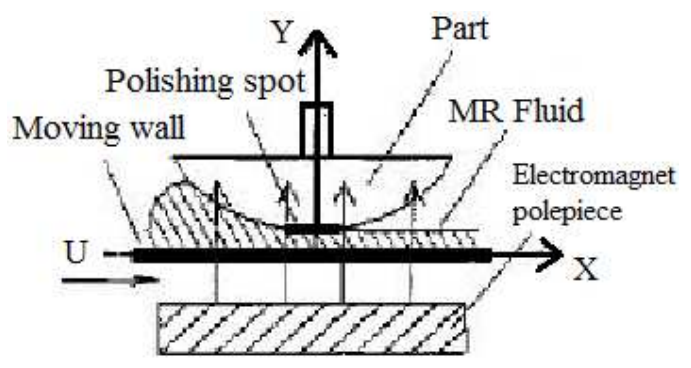

Figure 6. Schematic view of magneto-rheological polishing

In 1995, the optical processing centers of Rochester University made use of MR and processed a number of spherical and non-spherical optical elements that diameter is smaller than 50mm[22]. In 1997, they polished fused silica, glass material, and some types of infrared materials whose surface roughness is about $30 \mathrm{~nm}[23]$.

\section{Application in other Fields}

\subsection{MRF Sealing Device}

Kordonsky[24] studied the rotation shaft single-stage sealing technology by experiment where magneto-rheological fluid is sealed medium. It controls the viscosity of MRF in sealing gap to achieve the sealing purpose, its structure is consistent with the magnetic fluid sealing and has the advantages of simple structure, good sealing performance, no wear parts, easy maintenance, etc. Furthermore, Fujita[25] studied the effect of the magneto-rheological particle size, shape, and the viscosity of the carrier liquid on the rotational torque and the sealing pressure. Li Jingsong etc. [26] in Harbin Institute of Technology proposed a new MRF seal ring of low friction and long service life.

\subsection{MRF Composite Component}

In mechanical systems, such as plate, disc, beams, rods or other components, closing the MRF as a structural element in the relatively sandwich is a common practice to is a common practice to change the stiffness and damping of the composite components by changing the MRF shear and compression / tensile modulus. The composite components can be embedded in many mechanical systems to control the vibration and other properties of systems. Weiss[27] and Carlson[28] applied for a variety of composite component patents.

\subsection{MRF Flexible Fixture}

To solve the processing of small or irregularly shaped member in manufacturing process, using magneto-rheological flexible fixture can ensure the accuracy and reduce the processing time by making use of MRF 
characteristics of phase transition rapidly and the large yield strength. Tang[29] studied the possibility of manufacturing MRF flexible fixture and took the method of changing single-chain of microstructure into coarse columnar by pressure to meet the required of fixture carrying capacity in manufacturing processing.

\section{Conclusion}

Magnetorheological fluid is a new smart material and it is easy to control, under the action of an external magnetic field, it can achieve the transition between high yield stress viscoplastic colloid and Newtonian fluid in milliseconds and this transition is irreversible, continuous and controllable, so it has broad application prospects. This paper mainly introduces the composition of MRF, magneto-rheological principle and MRF application field. Domestic and foreign scholars achieved many achievements around the mechanism, testing, preparation, application and other aspects of MRF. But MRF technology and its applications still have many problems that need further research in following aspects: low performance of MRF; easy to leak, difficult to seal when working; poor particle sedimentation stability and instability; device cooling effect is not ideal; MRF control element, such as brake, clutch, output torque is not ideal, etc.. This technology will be widely used in the field of mechanical engineering after overcoming the above shortcomings.

\section{Acknowledgements}

This research was supported by the modern automobile service engineering subject platform construction in Shanghai City (XKCZ1214).

The first author Xu Biao was born in septemper.1990 in Anhui province. He got bachelor's degree of vehicle engineering in Anhui Polytechnic University in 2012 and now is a graduate student in Shanghai University of Engineering Science. Email:xubaio0813@163.com.

The second author Luo Yiping was born in 1966 . He gained a master's degree of Industrial Engineering in Shanghai Jiao Tong University in 1987. Now he is a professor in Shanghai University of Engineering Science.

The third author Ren Hongjuan was born in 1978 in Shandong province. She gained a master's degree of Power Machinery and engineering in Shandong University in 2002. Now she is an associate professor in Shanghai University of Engineering Science.

\section{References}

[1] Han Yiyan, He Guotian, Lin Yuanchang, etc.. Reviews on the magnetic particles of magnetorheological fluids[J]. Journal of function materials, 2013, 7(24): 3513-3519.

[2] Yang Jianjian, Yan Hua, Zhang Hui, etc.. Review and prospect of tribology study of magneto-rheological fluid[J]. Chemical industry and engineering progress, 2013, 32(8): 1855-1861.
[3] Christopher A. Waudby, John Christodoulou. GPU accelerated Monte Carlo simulation of pulsed-field gradient NMR experiments[J]. Journal of Magnetic Resonance, 2011, 211(1): 67-73.

[4] Kang S, Suh Y K. Direct simulation of flows with suspended paramagnetic particles using one-stage smoothed profile method[J]. Journal of Fluids and Structures, 2011, 27(2): 266-282.

[5] Arash Bahar, Francesc Pozo, Leonardo Acho, etc.. Parameter identification of large-scale magnetorheological dampers in a benchmark building $[J]$. Computers\&Structures, 2010, 88: 198-206.

[6] Kang Guiwen, Zhang Feihu. Surface shape control in optics manufacturing using magnetorheological finishing $[\mathrm{J}]$. Diamond\&Abrasives Enoneefing, 2010, 30(5): 77-79.

[7] Zhang Jinqiu, Zhang Jian, Kong Yanan, etc.. Summarization of Magnetorheological Fluid and Its Application[J]. Journal of Academy of Armored Force Engineering, 2010, 24(2): $1-6$.

[8] $\mathrm{Pu}$ Hongting, Jiang Fengjing. Research Progress and Application of Magnetorheological Fluids[J]. Chemical industry and engineering progress, 2005, 24(2): 132-136.

[9] Feng Weibang. Review on Magnetorheological Fluid Technology[J]. The ship electrical technology, 2012, 32(6): 15-18.

[10] Liu Cheng. Analysis and Design of Magnetorheological Fluid Brake[D]. Chongqing: Chongqing University of Science and Technology, 2010.

[11] Yang Guangqiang, Spencer B F. Large-scale magneto-rheological fluid damper for vibration mitigation: modeling, testing and control[D]. Indiana: Ph. D Dissertation University of Notre Dame, 2001.

[12] Faramarz Gordaninejad, Darrell G Breese. Magneto-rheological fluid damper[P]. US 2000, US Patent, No. 6019201. 2000.

[13] Li Zhongxian, Wu Linlin, Xu Longhe, etc.. Structural design of MR damper and experimental study for performance of damping force $[\mathrm{J}]$. Earthquake engineering and engineering vibration, 2003, 23 (1): 128-132.

[14] Wang Hongtao, Zhang Jinqiu, Bi Zhandong, etc.. Model and Analysis of Damping for Twin-tube Magneto-rheological Fluid Damper Based on Disc Type Orifice[J]. Journal of mechanical engineering, 2010, 46(18): 139-144.

[15] Wang Xiuyong, Sun Hongxin, Chen Zhengqing. Rotation shear magnetorheological fluid damper design and mechanical model[J]. Journal of vibration and shock, 2010, 29(10): 77-81.

[16] Lu He, Liu Xinhua. Magneto-rheological fluids and its applications in mechanical engineering $[\mathrm{J}]$. Technology and application of manufacturing, 2013, (1): 66-70.

[17] Zheng Jun, Zhang Guanghui, Cao Xingjin. Flow analysis of magnetorheological fluid in transmission device in steady state[J]. J. Cent. South Univ. (Science and Technology), 2008, 39(1): 149-154. 
[18] Hou Youfu, Tian Zuzhi, Wang Nannan. Numerical calculation of torque transmission and magnetic circuit finite element analysis of a magneto-rheological clutch[C]. CCIE 2010, 6: 403-407.

[19] Ding Baiqun, Song Yu. Structure Design and Performance Simulation for a Van Wheel Type Vehicle MRF Brake[J]. Automobile Technology, 2011, (8): 34-38.

[20] William Kordonsky. Elements and devices on magneto-rheological effect[J]. Journal of Intelligent Material Systems and Structures, 1993, 4(1): 65-69.

[21] W.M. I Kordonski, Adaptive Structures Base on Magnetorheological Fluids, Proc. 3 Int. Conf. Adaptive Struct., ed. Wada, Natori and Breitbach. P. 13-17, San Diego, CA, 1992.

[22] S.D. Jacobs, D. Golini, et al., Magnetorheological Finishing: A Deterministic Process for Optics Manufacturing, SPIE Vol. 2576, P. 372-382.

[23] S.D. Jacobs, et al., Magnetorheological Finishing of IR Materials, SPIE, Vol.3134, P. 258 269.
[24] Kordonaky W.I., Garodkin S.R., Magnetorheological Fluid Based Seal. In: Bullogh W.A., Proc of the 5th Int. Conf. on ER Fluids, MR Suspensions and Associated Technology, Singapore: World Scientific, 1996, P. 704-709.

[25] Fujita T, Jeysdevan B, Yoshimura K, et al., Characterization of MR Fluid for Seal. In: Tao T, Proc. of the 7th Int. Conf. on ER Fluids and MR Suspensions. Singapore: World Scientific, 2000, P. 721-727.

[26] Li Jingsong, Bowen. The magnetic fluid seal ring. Chinese patent: 00262392.7. 2001.

[27] Weiss K.D.,SuclosT.G., Chizan M.J., et al., Magnetorheological Fluid Composite Structures, U.S. Patent $5547049,1996$.

[28] Carlson, J.D. (1999). "Low-Cost MR Fluid Sponge Devices", Proc. 7th Int. Conf. on ERF, MR Suspensions \& Their Appli, Honolulu, Hawaii, July 19-23, ed. R. Tao.

[29] Tang $X$, Zhang $X$, Tao T. Flexible Fixture with Magnetorheological Fluids, In: Tao R, Proc. of the 7th Int. Conf. on ER Fluids and MR Suspensions. Singapore: World Scientific, 2000, P. 712-720. 